\title{
Effects of residential greenness on clinical outcomes of patients with chronic kidney disease: a large-scale observation study
}

\author{
Jae Yoon Park, ${ }^{1,2}$, Jiyun Jung ${ }^{3}$, Yong Chul Kim ${ }^{4}$, Hyewon Lee ${ }^{5}$, Ejin Kim ${ }^{6}$, Yon Su Kim, Ho Kim ${ }^{6,7}$, Jung Pyo Lee \\ ${ }^{1}$ Department of Internal Medicine, Dongguk University Ilsan Hospital, Ilsan, Republic of Korea \\ ${ }^{2}$ Department of Internal Medicine, Dongguk University College of Medicine, Gyeongju, Republic of Korea \\ ${ }^{3}$ Data Management and Statistics Institute, Dongguk University Ilsan Hospital, Ilsan, Republic of Korea \\ ${ }^{4}$ Department of Internal Medicine, Seoul National University College of Medicine, Seoul, Republic of Korea \\ ${ }^{5}$ Department of Health Administration and Management, College of Medical Sciences, Soonchunhyang University, Asan, Republic of Korea \\ ${ }^{6}$ Institute of Health and Environment, Seoul National University, Seoul, Republic of Korea \\ ${ }^{7}$ Department of Public Health Sciences, Graduate School of Public Health, Seoul National University, Seoul, Republic of Korea \\ ${ }^{8}$ Department of Internal Medicine, Seoul National University Boramae Medical Center, Seoul, Republic of Korea
}

Background: As industrialization and urbanization are accelerating, the distribution of green areas is decreasing, particularly in developing countries. Since the 2000s, the effects of surrounding greenness on self-perceived health, including physical and mental health, longevity, and obesity have been reported. However, the effects of surrounding green space on chronic kidney disease are not well understood. Therefore, we investigated the impact of residential greenness on the mortality of chronic kidney disease patients and progression from chronic kidney disease to end-stage renal disease (ESRD).

Methods: Using a large-scale observational study, we recruited chronic kidney disease patients ( $n=64,565$; mean age, 54.0 years; $49.0 \%$ of male) who visited three Korean medical centers between January 2001 and December 2016. We investigated the hazard ratios of clinical outcomes per 0.1-point increment of exposure to greenness using various models.

Results: During the mean follow-up of $6.8 \pm 4.6$ years, 5,512 chronic kidney disease patients developed ESRD (8.5\%) and 8,543 died (13.2\%). In addition, a 0.1-point increase in greenness reduced all-cause mortality risk in chronic kidney disease and ESRD patients and progression of chronic kidney disease to ESRD in a fully adjusted model. The association between mortality in ESRD patients and the normalized difference vegetation index was negatively correlated in people aged >65 years, who had normal weight, were nonsmokers, and lived in a nonmetropolitan area.

Received: November 16, 2020; Revised: February 15, 2021; Accepted: February 28, 2021

Editor: Woo Yeong Park, Keimyung University, Daegu, Republic of Korea

Correspondence: Jung Pyo Lee

Department of Internal Medicine, Seoul National University Boramae Medical Center, 20 Boramae-ro 5-gil, Dongjak-gu, Seoul 07061, Republic of Korea. E-mail: nephrolee@gmail.com

ORCID: https://orcid.org/0000-0002-4714-1260

Ho Kim

Department of Public Health Sciences, Graduate School of Public Health, Seoul National University, 1 Gwanak-ro, Gwanak-gu, Seoul 08826,

Republic of Korea. E-mail: hokim@snu.ac.kr

ORCID: https://orcid.org/0000-0001-7472-3752

Jae Yoon Park and Jiyun Jung contributed equally to this work as co-first authors.

Copyright (C) 2021 by The Korean Society of Nephrology

(a) This is an Open Access article distributed under the terms of the Creative Commons Attribution Non-Commercial and No Derivatives License (http:// creativecommons.org/licenses/by-nc-nd/4.0/) which permits unrestricted non-commercial use, distribution of the material without any modifications, and reproduction in any medium, provided the original works properly cited. 
Conclusion: Chronic kidney disease patients who live in areas with higher levels of greenness are at reduced risk of all-cause mortality and progression to ESRD.

Keywords: Chronic kidney disease, End-stage renal disease, Normalized difference vegetation index, Residential greenness

\section{Introduction}

According to the World Health Organization's report in 2016, $23 \%$ of all deaths worldwide (around 12.6 million) were attributable to environmental factors (e.g., air pollutants), and these factors accounted for $22 \%$ of the global burden of disease [1]. As industrialization and urbanization are accelerating, the distribution of green areas is decreasing, particularly in developing countries. Since the 2000s, the effects of surrounding greenness on self-perceived health, including physical (e.g., fatigue) and mental health (e.g., mood), longevity, and obesity have been reported [2]. Greenness has been hypothesized to benefit health by reducing exposure to air pollution, extreme heat, and noise, providing opportunities for physical activity and social engagement, and decreasing psychological stress and depression through direct contact with nature [3]. In recent years, it has been reported that a decrease in green areas adversely affects specific clinical outcomes, such as mortality due to cardiovascular (CV) disease, respiratory disease, and kidney disease, as well as birth outcomes and mental and physical health.

Chronic kidney disease (CKD) has become a critical public health issue worldwide, with a steadily increasing prevalence and poor clinical outcomes, and it imposes a high medical cost burden. Approximately 26 million adults in the United States and 4.6 million adults in Korea have CKD [4], and the annual per-person medical cost attributable to CKD was $\$ 1,700$ for stage $2, \$ 3,500$ for stage 3 , and $\$ 12,700$ for stage 4 [5]. Individuals with CKD are 8- to 10-fold more likely to have CV mortality than those without renal dysfunction [6]. The traditional risk factors for CKD are diabetes mellitus (DM), hypertension, dyslipidemia, smoking, old age, and male sex [7]. Although the management of these factors has advanced considerably, the risk of CKD and its complications remain significant.

Accordingly, it is important to investigate and control new threats as well as traditional risk factors in order to establish a new perspective on CKD management. Recent reports have provided evidence that environmental factors, such as air pollution and sunlight exposure, also play important roles in kidney disease [8]. However, there is limited evidence for the impact of greenness on CKD patients.

Therefore, we have investigated the impact of residential greenness on mortality in CKD and end-stage renal disease (ESRD) patients and on disease progression from CKD to ESRD using a large-scale observational cohort from multiple metropolitan hospitals in Korea.

\section{Methods}

\section{Study population}

A cohort of 66,492 patients who visited three medical centers (Seoul National University Hospital, Seoul National University Bundang Hospital, and Seoul National University Boramae Medical Center) in Korea were followed from January 2001 to December 2016. We defined CKD patients according to the 2012 Kidney Disease: Improving Global Outcomes (KDIGO) Clinical Practice Guideline for the Evaluation and Management of CKD report [9]. The guideline defined CKD as abnormalities of kidney structure or function which were present for $>3$ months with implications for health. We excluded those whose residences were unclear $(\mathrm{n}=7)$ and who were followed up for less than 3 months ( $\mathrm{n}$ $=1,920$ ). Therefore, 64,565 CKD patients were eventually enrolled in the study. We investigated the effect of greenness on three types of clinical outcomes; all-cause mortality in CKD patients, ESRD development in CKD patients, and allcause mortality in ESRD patients. The date of death was confirmed from Statistics Korea. During follow-up, the date of ESRD outcome is selected as the earliest of the dates when the diagnostic code was entered, the dialysis prescription was first ordered, or when surgery for dialysis access (arteriovenous fistula or catheter insertion for peritoneal dialysis) was performed. 


\section{Ethical aspects}

The study complied with the Declaration of Helsinki and received full approval from the Institutional Review Board of Seoul National University Hospital (No. J-1704-121848), Seoul National University Bundang Hospital (No. B-1706/401-402), and Seoul National University Boramae Medical Center (No. 20170414/16-2017-65/051). The written informed consent waiver was also approved.

\section{Normalized difference vegetation index}

Green space was defined using the normalized difference vegetation index (NDVI) derived from the Moderate-Resolution Image Spectroradiometer (MODIS) Terra satellite images, which are generated every 16 days at $250-\mathrm{m}$ spatial resolution by NASA [10]. Previous studies have used NDVI as a representative indicator of green space [11]. The NDVI is calculated as the ratio of the sum and the difference between red and near-infrared (NIR) light (NDVI = [NIR - Red]/[NIR + Red]), based on fact that the mesophyll leaf structure reflects NIR, whereas chlorophyll in plants absorbs the red frequencies of visible light [12]. It ranges from -1 to 1 , with higher positive values indicating dense vegetation areas and higher negative values representing bare soil, water, and snow [13]. Patients' exposure to vegetation was estimated using the average NDVI in summer (June-August) within a radius of $250 \mathrm{~m}$ and 1,250 $\mathrm{m}$ around participants' residences. We assigned the summer NDVI with high vegetation at 1 year before cohort enrollment as an estimate of maximum exposure [14]. NDVI within $250 \mathrm{~m}$ and 1,250 $\mathrm{m}$ around the participants' residences in the year before their cohort entry was used to investigate the long-term effects, as NDVI measured from MODIS images was available from 2000. Measurement within a radius of $250 \mathrm{~m}$ indicated the directly accessible greenness, whereas vegetation within a radius of 1,250 $\mathrm{m}$ around each patient's residence referred to greenness accessible within a 10- to 15-minute walkable distance [15].

\section{Covariates}

The following covariates were included: age at baseline, sex (one, male; two, female), hemoglobin, hypertension, DM, estimated glomerular filtration rate (eGFR), average concen- tration of particulate matter less than $10 \mu \mathrm{m}\left(\mathrm{PM}_{10}\right)$, population density, financial independence rate, and number of hospital beds. We calculated eGFR as follows [16]:

$$
\text { eGFR } \left.=175 \times \text { Creatinine }^{-1.154} \times \text { Age }^{-0.203} \times 0.742 \text { (if female }\right)
$$

Subjects were defined as having hypertension if they were prescribed antihypertensive medications, were registered with diagnostic codes for hypertension on the electronic medical chart, and met the following hypertension criteria at the time of blood pressure measurement; systolic blood pressure $\geq 140 \mathrm{mmHg}$ or diastolic blood pressure $\geq 90$ mmHg. In addition, subjects were defined as having DM if they were being prescribed DM medication, or were registered with diagnostic codes for DM on the electronic medical chart at the time of enrollment. The hourly $\mathrm{PM}_{10}$ was obtained from 274 monitoring stations nationally through the Urban Atmospheric Monitoring Network between 2000 and 2016. Average $\mathrm{PM}_{10}$ values of monitoring stations within $3 \mathrm{~km}, 5 \mathrm{~km}$, and $10 \mathrm{~km}$ radii around participants' residences were used, since the monitoring stations are not distributed equally throughout the country. Therefore, $\mathrm{PM}_{10}$ was not assigned if there was no observatory within $3 \mathrm{~km}(\mathrm{n}=15,798)$, $5 \mathrm{~km}(\mathrm{n}=7,365)$, and $10 \mathrm{~km}(\mathrm{n}=4,172)$ from the residence. In addition, the mean concentration of $\mathrm{PM}_{10}$ was updated every year, from 1 year before entry into the cohort, to reflect the time variance in this parameter. We included geographic characteristics by examining the population density, financial independence rate, and the number of hospital beds in the districts of each participant's residence.

\section{Statistical analyses}

We applied Cox proportional hazard models to estimate the hazard ratios (HRs) and their 95\% confidence intervals (CIs) for the associations between 0.1-point increases in residential greenness (NDVI) and clinical outcomes in four types of models. (1) Model 1 was a crude model that analyzed the effects of NDVI. (2) Model 2 was additionally stratified by 5 -year age groups from 20 to 90 years of age and was adjusted for sex, eGFR, hemoglobin, hypertension, and DM. (3) Model 3 was additionally modified by $\mathrm{PM}_{10}$ within $3 \mathrm{~km}, 5$ $\mathrm{km}$, and $10 \mathrm{~km}$ around patients' residences. (4) Model 4 (fully adjusted model) was additionally adjusted for geographical variables, such as population density, financial indepen- 
dence rate, and the number of hospital beds. In addition, we stratified all models by the entrance year into the cohort, to reflect the different cohort entries of participants. We used Schoenfeld residuals to test whether the hazard rate was constant in time by the proportional hazard assumption. Tests for nonlinearity were conducted using the ordinal for each quartile. Observation periods were calculated from the entrance into the cohort, censored at the date of clinical outcomes or the end of follow-up (December 31, 2016). We conducted stratified analysis by potential confounders: urbanity, smoking, alcohol (yes vs. no), body mass index (BMI, $<25.0 \mathrm{~kg} / \mathrm{m}^{2}$ vs. $\geq 25.0 \mathrm{~kg} / \mathrm{m}^{2}$ ), and age ( $<65$ years vs. $\geq 65$ years). Urban environment included seven major cities in Korea (Seoul, Busan, Incheon, Daegu, Daejeon, Gwangju, and Ulsan) with over 1 million people. All analyses were conducted using R software (ver. 3.4.3; R Foundation for Statistical Computing, Vienna, Austria).

\section{Results}

Supplementary Fig. 1 (available online) shows the flow chart of 64,565 participants in the cohort. Table 1 presents the baseline characteristics of the study population with several exclusions in Supplementary Fig. 1. The average age was 54.0 years, and $49.0 \%$ of patients were male. Those who were living in the highest quartile of the 250-m NDVI tended to be slightly older, had lower hemoglobin levels, air pollutants, and financial independence rates, and more likely to live in nonmetropolitan areas compared to those living in the lowest 250-m NDVI quartile. During a mean follow-up period of 6.8 years, 8,546 deaths occurred in the cohort $(13.2 \%)$ and 5,512 CKD patients developed ESRD (8.5\%). The mortality rate of ESRD patients was $23.5 \%(n=1,918)$. The mean eGFR of participants was $62.6 \mathrm{~mL} / \mathrm{min} / 1.73 \mathrm{~m}^{2}$. In addition, more than half of the CKD patients (55.0\%) lived in metropolitan areas. The mean concentration of $\mathrm{PM}_{10}$ was measured within $3 \mathrm{~km}, 5 \mathrm{~km}$, and $10 \mathrm{~km}$. Fig. 1 shows the NDVI distribution in Korea, in June, from 2001 to 2015. The green environment had improved substantially by 2015 as compared to 2001 . The average summer residential greenness before a year of enrollment, within $250 \mathrm{~m}$ and 1,250 $\mathrm{m}$ of the participant's residence, was 0.38 and 0.41 , respectively. There was a greener environment within a 10- to 15 -minutes walkable distance from patients' residences than near their homes (Supplementary Table 1, available online). Participants in the cohort resided throughout the country, including the islands in the west, Ulleungdo in the east, and Jeju Island in the south. Supplementary Fig. 2 provides the regional distribution of all-cause mortality in CKD patients, ESRD development in CKD patients, and all-cause mortality in ESRD patients; most participants with adverse outcomes were concentrated in dense population areas.

We investigated the HRs of clinical outcomes per 0.1-point increment of exposure to greenness using various models (Supplementary Table 2, available online). In model 1, every 0.1-point increase in NDVI within both the $250 \mathrm{~m}$ and 1,250 $\mathrm{m}$ radii was associated with an increased risk of all-cause mortality and occurrence of ESRD in CKD patients. However, we found protective effects of green space on the mortality risk of ESRD patients in the crude model. After adjustment for individual-level covariates (model 2), the increased risk effects of NDVI were reduced. HRs by 0.1-point NDVI increase in green space within the $1,250 \mathrm{~m}$ and $250 \mathrm{~m}$ were 0.98 (95\% CI, 0.96-1.01) for mortality and 1.00 (95\% CI, 0.98-1.02) for progression to ESRD, respectively. The impact of a green environment on reducing mortality risk and progression to ESRD was stronger when the model was additionally adjusted for the mean concentration of $\mathrm{PM}_{10}$, regardless of radius.

In the fully adjusted model, we found a consistent association between both continuous values and quartiles of greenness and clinical outcomes (Table 2). Patients living in the highest quartile of greenness according to the 1,250 $m$ radius CKD and ESRD patients had a $1.1 \%(0 \%-2.3 \%)$ and $2.3 \%(0.4 \%-4.1 \%)$ lower mortality risk, respectively than those living in the lowest greenness quartile. In addition, CKD patients living in the highest quartile of NDVI, according to the 250-m radius, showed less frequent progression to ESRD than those living in the lowest NDVI quartile (HR, 0.996; 95\% CI, 0.99-1.01). We confirmed the linear trend of association between mortality and NDVI (p-values of 0.0060.046). Greenness within $1,250 \mathrm{~m}$ had significant protective effects against mortality in CKD patients (HR, 0.96; 95\% CI, 0.93-0.996) and ESRD patients (HR, 0.91; 95\% CI, 0.87-0.97). The HR for each 0.1-point increase in NDVI in the 250-m radius on progression to ESRD was 0.98 (95\% CI, 0.95-1.01).

Table 3 shows the results of stratified analyses by model 4 , which was adjusted for potential confounders such as urbanity, smoking, alcohol, age, and BMI. Strong protective effects of both a 250-m and 1,250-m buffer of greenness in terms of reducing mortality risk were seen in CKD patients 
Table 1. Baseline characteristics of the study population $(n=64,565)$ according to the quartile of the NDVI within a $250-m$ radius

\begin{tabular}{|c|c|c|c|c|c|}
\hline Characteristic & Total & Quartile 1 & Quartile 2 & Quartile 3 & Quartile 4 \\
\hline \multicolumn{6}{|l|}{ NDVI } \\
\hline $250 \mathrm{~m}$ & $0.38 \pm 0.15$ & $0.22 \pm 0.03$ & $0.31 \pm 0.02$ & $0.41 \pm 0.04$ & $0.60 \pm 0.09$ \\
\hline Age (yr) & $54.0 \pm 17.0$ & $53.3 \pm 16.7$ & $54.0 \pm 17.2$ & $54.4 \pm 17.2$ & $54.4 \pm 16.9$ \\
\hline \multicolumn{6}{|l|}{ Sex } \\
\hline Male & 31,613 (49.0) & $7,580(47.0)$ & $7,933(49.2)$ & 7,889 (48.9) & $8,206(50.8)$ \\
\hline \multicolumn{6}{|l|}{ Body mass index $\left(\mathrm{kg} / \mathrm{m}^{2}\right)$} \\
\hline$<25$ & $28,079(43.5)$ & 6,350 (39.3) & $7,183(44.5)$ & $7,332(45.4)$ & $7,211(44.7)$ \\
\hline$\geq 25$ & $13,676(21.2)$ & $2,983(18.5)$ & 3,398 (21.1) & $3,594(22.3)$ & 3,699 (22.9) \\
\hline Missing & $22,810(35.3)$ & $6,806(42.2)$ & $5,558(34.4)$ & $5,213(32.3)$ & $5,230(32.4)$ \\
\hline Observation period (yr) & $6.8 \pm 4.5$ & $8.1 \pm 4.7$ & $6.5 \pm 4.4$ & $6.1 \pm 4.2$ & $6.2 \pm 4.2$ \\
\hline \multicolumn{6}{|l|}{ Diabetes mellitus } \\
\hline Yes & $14,239(22.1)$ & $3,466(21.5)$ & $3,411(21.1)$ & $3,599(22.3)$ & $3,761(23.3)$ \\
\hline No & 50,326 (77.9) & $12,673(78.5)$ & $12,728(78.9)$ & $12,540(77.7)$ & $12,379(76.7)$ \\
\hline eGFR $\left(\mathrm{mL} / \mathrm{min} / 1.73 \mathrm{~m}^{2}\right)$ & $62.6 \pm 31.9$ & $64.0 \pm 29.1$ & $63.4 \pm 33.4$ & $62.0 \pm 32.0$ & $61.2 \pm 32.6$ \\
\hline Hemoglobin (g/dL) & $12.9 \pm 2.2$ & $13.1 \pm 2.2$ & $12.9 \pm 2.2$ & $12.9 \pm 2.2$ & $12.8 \pm 2.2$ \\
\hline \multicolumn{6}{|l|}{ Smoking } \\
\hline Yes & $3,321(5.1)$ & $869(5.4)$ & $926(5.7)$ & $781(4.8)$ & $744(4.6)$ \\
\hline No & $27,427(42.5)$ & $8,622(53.4)$ & $7,792(48.3)$ & $6,067(37.6)$ & $4,944(30.6)$ \\
\hline Missing & $33,817(52.4)$ & $6,648(41.2)$ & $7,421(46.0)$ & $9,291(57.6)$ & $10,452(64.8)$ \\
\hline \multicolumn{6}{|l|}{ Alcohol } \\
\hline $10 \mathrm{~km}$ & $57.2 \pm 11.2$ & $60.2 \pm 12.6$ & $56.2 \pm 10.9$ & $55.7 \pm 9.9$ & $56.4 \pm 10.3$ \\
\hline Metropolitan resident & $35,528(55.0)$ & $14,548(90.1)$ & $10,926(67.7)$ & $6,824(42.3)$ & $3,230(20.0)$ \\
\hline Population density (person $/ \mathrm{km}^{2}$ ) & $11,064 \pm 8,265$ & $16,481 \pm 6,599$ & $13,125 \pm 7,692$ & $9,380 \pm 7,847$ & $5,124.64 \pm 6,389$ \\
\hline Financial independence rate (\%) & $48.5 \pm 18.6$ & $48.2 \pm 18.2$ & $46.6 \pm 17.9$ & $47.4 \pm 17.8$ & $45.3 \pm 19.1$ \\
\hline The number of hospital beds & $2,596 \pm 1,273$ & $2,773 \pm 1,460$ & $2,665 \pm 1,209$ & $2,514 \pm 1,123$ & $2,198 \pm 1,096$ \\
\hline
\end{tabular}

Data are expressed as mean \pm standard deviation or number (\%).

eGFR, estimated glomerular filtration rate; NDVI, normalized difference vegetation index; $\mathrm{PM}_{10}$, particulate matter less than $10 \mu \mathrm{m}$.

older than 65 years. In the association between greenness and progression from CKD to ESRD, we observed inverse HRs in nonmetropolitan residents as compared to metropolitan residents, but the differences between groups were not significant ( $\mathrm{p}$ for interaction $=0.44$ ). A green environment within a 1,250-m radius reduced the mortality risk of ESRD patients in an urbanity-, smoking-, age-, and BMI-specific association. We found stronger protective effects of greenness in patients with normal weight who lived in metropolitan areas than in individuals with BMI over $25.0 \mathrm{~kg} / \mathrm{m}^{2}$ living in nonmetropolitan areas, although there was no significant difference between these groups ( $\mathrm{p}$ for interaction $=0.43-0.46$ ). When stratified by age, exposure to greenness had positive effects in people older than 65 years (HR, 0.89; 

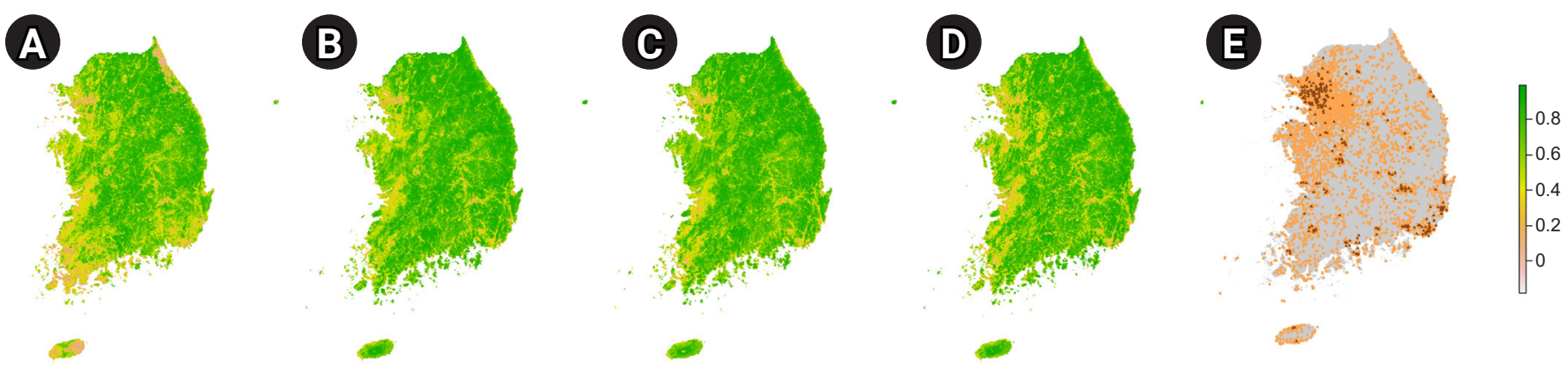

Figure 1. Normalized difference vegetation index distribution in Korea. In June 2001 (A), 2005 (B), 2010 (C), 2015 (D), and (E) the regional distribution of participants ( $n=64,565)$, with monitoring stations for mean particulate matter less than $10 \mu \mathrm{m}$ concentration (triangle).

Table 2. Hazard ratio (95\% confidence interval) for clinical outcomes by the NDVI within a radius of $250 \mathrm{~m}$ and of $1,250 \mathrm{~m}$ around the residences of CKD patients in the fully adjusted model

\begin{tabular}{cccccccc}
\hline Variable & NDVI buffer & Quartile 1 & Quartile 2 & Quartile 3 & Quartile 4 & p for trend & Continuous $^{\mathrm{b}}$ \\
\hline CKD patient & & & & & & \\
Mortality & $250 \mathrm{~m}$ & Reference & $1.00(0.99-1.00)$ & $0.99(0.98-1.00)$ & $0.99(0.98-1.00)$ & 0.0458 & $0.97(0.94-1.00)$ \\
& $1,250 \mathrm{~m}$ & Reference & $1.00(0.99-1.00)$ & $0.99(0.98-1.00)$ & $0.99(0.98-1.00)$ & 0.0255 & $0.96(0.93-1.00)$ \\
ESRD & $250 \mathrm{~m}$ & Reference & $1.00(0.99-1.01)$ & $1.01(1.00-1.02)$ & $1.00(0.99-1.01)$ & 0.6603 & $0.98(0.95-1.01)$ \\
& $1,250 \mathrm{~m}$ & Reference & $1.00(0.99-1.01)$ & $1.01(1.00-1.02)$ & $1.00(0.99-1.01)$ & 0.4745 & $1.01(0.98-1.04)$ \\
ESRD patient & & & & & & \\
Mortality & $250 \mathrm{~m}$ & Reference & $1.00(0.98-1.01)$ & $0.99(0.97-1.00)$ & $1.00(0.98-1.01)$ & 0.3963 & $0.98(0.93-1.03)$ \\
& $1,250 \mathrm{~m}$ & Reference & $0.98(0.96-0.99)$ & $0.97(0.96-0.99)$ & $0.98(0.96-1.00)$ & 0.0059 & $0.91(0.87-0.97)$ \\
\hline
\end{tabular}

Multivariable fully adjusted model included covariates of age(5-year age groups from 20 to 90 years), sex, hypertension, diabetes, hemoglobin, estimated glomerular filtration ratio, average concentration of PM10 monitoring stations within $5 \mathrm{~km}$ around the residence, population density, financial independence rate, and the number of hospital beds.

CKD, chronic kidney disease; ESRD, end-stage renal disease; NDVI, normalized difference vegetation index.

${ }^{a}$ Test for linear trend using the ordinal for each quartile; ${ }^{b} 0.1$ unit increase.

95\% CI, 0.82-0.96), although the difference between groups was not significant $(\mathrm{p}=0.24)$. We also confirmed that nonsmokers had a greater protective effect of greenness against progression to ESRD compared to smokers.

\section{Discussion}

In this study, we evaluated the long-term effects of NDVI on mortality and progression to ESRD in patients with CKD in Korea between 2001 and 2016. Participants with CKD and ESRD exposed to a green space within a walkable distance of 10 to 15 minutes had markedly reduced mortality risks, while the risk of progression from CKD to ESRD was also significantly decreased by living within an area with directly accessible green space. We found that the benefits of residential greenness on mortality were greater in metropolitan residents who were 65 years or older with a healthy lifestyle (as indicated by nonsmoking and normal weight).

Previously, several studies reported a lower mortality risk among people living in greener areas. An 11-year study using a large cohort of individuals living in Canada reported that a 0.15 -point increase in NDVI around participants' residences reduced non-accidental, CV disease-, and respiratory disease-related deaths by $8.5 \%, 8.9 \%$, and $10.1 \%$, respectively [17]. In addition, US women living in the highest quintile of greenness within a $250-\mathrm{m}$ radius had $12 \%, 13 \%$, and $34 \%$ lower risk of all-cause non-accidental, cancer-related, and respiratory disease-related mortality, respectively, than those living in the lowest quintile [15]. Several studies have also reported a protective effect of greenness against infant mortality [18], anxiety and depression, and impaired fetal growth [19]. However, a meta-analysis of six studies conducted in 2016, which estimated the association between all-cause mortality and greenness showed nonsignificant 
Table 3. Stratified hazard ratio (95\% confidence interval) for clinical outcomes by the NDVI ${ }^{\mathrm{a}}$

\begin{tabular}{|c|c|c|c|c|c|c|}
\hline \multirow{3}{*}{ Variable } & \multicolumn{4}{|c|}{ CKD patient } & \multirow{2}{*}{\multicolumn{2}{|c|}{$\begin{array}{c}\text { ESRD patient } \\
\text { Mortality }\end{array}$}} \\
\hline & \multicolumn{2}{|c|}{ Mortality } & \multicolumn{2}{|c|}{ ESRD } & & \\
\hline & NDVI $250 \mathrm{~m}$ & NDVI 1,250 m & NDVI $250 \mathrm{~m}$ & NDVI 1,250 m & NDVI $250 \mathrm{~m}$ & NDVI 1,250 m \\
\hline \multicolumn{7}{|c|}{ Urbanicity } \\
\hline No & $1.05(0.99-1.10)$ & $1.06(1.00-1.13)$ & $0.96(0.93-1.00)$ & $0.99(0.94-1.04)$ & $1.00(0.93-1.08)$ & $0.94(0.86-1.03)$ \\
\hline Yes & 0.97 (0.92-1.01) & $0.97(0.92-1.03)$ & $0.98(0.94-1.03)$ & $1.00(0.96-1.05)$ & $0.97(0.90-1.05)$ & $0.91(0.83-0.99)$ \\
\hline $\mathrm{p}_{\text {interact }}$ & 0.35 & 0.97 & 0.44 & 0.49 & 0.56 & 0.46 \\
\hline \multicolumn{7}{|l|}{ Smoking } \\
\hline No & $0.98(0.94-1.03)$ & $0.98(0.93-1.04)$ & 0.99 (0.96-1.03) & $1.01(0.97-1.06)$ & $0.99(0.92-1.06)$ & $0.90(0.84-0.97)$ \\
\hline Yes & $1.12(0.99-1.26)$ & $1.09(0.95-1.25)$ & $1.00(0.90-1.11)$ & $1.06(0.94-1.20)$ & $1.02(0.82-1.26)$ & $1.05(0.83-1.33)$ \\
\hline $\mathrm{p}_{\text {interact }}$ & 0.05 & 0.04 & 0.86 & 0.91 & 0.11 & 0.02 \\
\hline \multicolumn{7}{|l|}{ Alcohol } \\
\hline No & $1.00(0.96-1.05)$ & $1.00(0.95-1.05)$ & $1.00(0.96-1.04)$ & $1.02(0.98-1.06)$ & $1.00(0.94-1.07)$ & $0.93(0.87-1.00)$ \\
\hline Yes & $1.03(0.91-1.16)$ & $1.07(0.94-1.22)$ & $0.92(0.82-1.03)$ & $0.99(0.87-1.12)$ & $1.10(0.84-1.46)$ & $1.12(0.81-1.55)$ \\
\hline $\mathrm{p}_{\text {interact }}$ & 0.23 & 0.04 & 0.56 & 0.77 & 0.54 & 0.62 \\
\hline \multicolumn{7}{|l|}{ Age (yr) } \\
\hline$<65$ & 1.03 (0.98-1.09) & $1.00(0.94-1.06)$ & $0.98(0.94-1.01)$ & $1.01(0.97-1.05)$ & $1.00(0.94-1.08)$ & $0.94(0.86-1.01)$ \\
\hline$\geq 65$ & $0.94(0.90-0.98)$ & $0.95(0.91-0.99)$ & $0.99(0.94-1.04)$ & $1.02(0.96-1.07)$ & $0.95(0.89-1.02)$ & $0.89(0.82-0.96)$ \\
\hline $\mathrm{p}_{\text {interact }}$ & 0.001 & 0.045 & 0.52 & 0.72 & 0.22 & 0.24 \\
\hline \multicolumn{7}{|c|}{ Body mass index $\left(\mathrm{kg} / \mathrm{m}^{2}\right)$} \\
\hline$<25$ & $0.97(0.93-1.02)$ & $0.96(0.92-1.01)$ & $0.98(0.95-1.01)$ & $1.01(0.97-1.04)$ & $0.96(0.90-1.02)$ & $0.90(0.84-0.96)$ \\
\hline$\geq 25$ & 0.99 (0.91-1.07) & $1.01(0.92-1.10)$ & $1.01(0.95-1.08)$ & $1.04(0.97-1.11)$ & $0.99(0.86-1.14)$ & $0.92(0.79-1.08)$ \\
\hline $\mathrm{p}_{\text {interact }}$ & 0.19 & 0.08 & 0.39 & 0.43 & 0.28 & 0.43 \\
\hline
\end{tabular}

Multivariable fully adjusted model included covariates of age(5-year age groups from 20 to 90 years), sex, hypertension, diabetes, hemoglobin, estimated glomerular filtration ratio, average concentration of PM10 monitoring stations within $5 \mathrm{~km}$ around the residence, population density, financial independence rate, and the number of hospital beds.

CKD, chronic kidney disease; ESRD, end-stage renal disease; NDVI, normalized difference vegetation index; $P_{\text {interact }}$ p for interaction between stratification variable and NDVI.

${ }^{a} \mathrm{NDVI} 0.1$ unit increase

results when greenness increased by 0.1 units (relative risk, 0.992; 95\% CI, 0.98-1.01) [20]. However, the results of the study were not generalizable, as this meta-analysis was performed using a relatively small number of studies.

In recent years, studies that explored the relationship between residential greenness and major causes of CKD, such as DM, hypertension, obesity, and dyslipidemia, have been reported. In 2014, the United Kingdom researchers reported that the risk of DM, the most common cause of CKD, was inversely associated with green space [21]. More recently, studies from China also reported that the risk of DM was reduced by greenness, which was measured using the NDVI [22]. The risk of hypertension, the second most important cause of CKD, has been consistently reported to be decreased by a green environment since the 2000s. The risk of metabolic syndrome, which involves obesity and dyslipidemia, has also been reported to be decreased in green spaces [23]. The specific mechanisms of the relationship between greenness and the major risk factors of CKD are not fully understood, but the reports commonly mentioned that green space would reduce ambient air pollution [24]. Ambient air pollutants, such as nitric oxide, $\mathrm{PM}_{10}$, and $\mathrm{PM}_{2.5}$, have been reported as risk factors for DM in several studies [25]. Air pollution also has been reported as a risk factor for hypertension, obesity, and dyslipidemia [26]. Other general positive effects of greenness included increased physical activity, social cohesion, and microbial exposure and decreased psychological stress, air temperature levels, exposure to volatile organic compounds, and noise [27].

In subgroup analysis, our results demonstrated that green space could reduce mortality in CKD and ESRD patients over 65 years of age. While previous reports did not reveal a significant association between age and greenness [28], at the beginning of 2020, studies from China reported more 
significant protective effects of greenness against metabolic syndrome in participants aged under 65 years [29]. The most problematic issue in the elderly population is frailty, which is a condition in which various physiological functions have deteriorated due to age. These individuals are vulnerable to sudden health problems caused by even minor stress events [30]. Our findings are consistent with frailty in elderly people. We also observed significant effects of greenness on subpopulations with BMI less than $25.0 \mathrm{~kg} / \mathrm{m}^{2}$ who do not consume alcohol or smoke. These results indicate that the effect of greenness was more significant in CKD patients with well-controlled modifiable risk factors. Consequently, the results could suggest that deficiency of residential greenness is a new risk factor, in addition to well-identified, modifiable risk factors for CKD, such as obesity, alcohol, and smoking.

We found that greenness affects not only mortality but also progression of CKD to ESRD. Although statistically significant associations were not shown in the main analysis, greenness reduced ESRD occurrence significantly in patients living in nonmetropolitan areas, indicating that a lack of green space contributes to progression of kidney disease, as well as to mortality in those with kidney disease. Moreover, our findings are consistent with previous studies showing that representative risk factors for $\mathrm{CKD}$, such as $\mathrm{DM}$, hypertension, obesity, and dyslipidemia, could be modified positively by greenness.

Our study has several strengths. First, no previous study has reported a direct examination of the effect of greenness on mortality in CKD patients. Second, we adjusted for the comorbidities of DM and hypertension, biochemical indices that are important for predicting mortality risk, and socioeconomic factors, such as population density, financial independence rate, and the number of hospital beds. In addition, we estimated the greenness effect by modifying models by the time-varied measurement of particulate matter concentrations. Third, no previous study has reported the effects of greenness on progression of CKD to ESRD.

This study had the following limitations. First, we obtained data based on residence at entry into the cohort, and changes in residence were not updated. NDVI values reflecting address relocation could provide more accurate exposure measures. Second, although the cohort data contained information on education and socioeconomic status in terms of three levels (low, middle, and high), this could not be used as a modifier in the main or stratified analyses because the response rate for these variables were $35.5 \%$ and $9.0 \%$, respectively. In addition, relevant individual risk factors, such as physical activity and social engagement, which are considered as modulators [31], were not available for this cohort. A study conducted in China found that adults 65 years or older, living in the highest quartile of residential greenness, scored $28 \%$ more on activities of daily living than those living in the lowest quartile [32]. Thus, our study did not explore how much participants utilized the green space for exercise and relaxation. Third, unmeasured confounding factors of area-level socioeconomic status could have affected the association between greenness and clinical outcomes in CKD patients.

In conclusion, we report that greenness surrounding patients' residences reduced the risk of mortality in CKD and ESRD patients and the progression from CKD to ESRD. This study suggests that a deficiency in residential greenness is a new modifiable risk factor for CKD.

\section{Conflicts of interest}

All authors have no conflicts of interest to declare.

\section{Funding}

This work was supported by the Seoul National University Research Grant in 2018, the Korean Society of Nephrology Grant in 2018 (BAXTER), and the Basic Science Research Program through the National Research Foundation of Korea (NRF) funded by the Ministry of Education (2020R1A6A3A01099724).

\section{Acknowledgments}

The data used in this study were obtained from Seoul National University Hospital and Seoul National University Boramae Medical Center in Seoul and Seoul National University Bundang Hospital in Seongnam, Republic of Korea.

\section{Authors' contributions}

Conceptualization: HK, JPL

Data curation: YCK

Formal analysis: JJ

Funding acquisition: HK, JPL, JJ

Investigation, Visualization: JYP, JJ 
Methodology: HL, EK

Project administration: YSK, HK, JPL

Writing-original draft: JYP, JJ

Writing-review \& editing: All authors

All authors read and approved the final manuscript.

\section{ORCID}

Jae Yoon Park, https://orcid.org/0000-0001-8986-7492

Jiyun Jung, https://orcid.org/0000-0002-8235-0316

Yong Chul Kim, https://orcid.org/0000-0003-3215-8681

Hyewon Lee, https://orcid.org/0000-0002-6041-0840

Ejin Kim, https://orcid.org/0000-0001-6293-785X

Yon Su Kim, https://orcid.org/0000-0003-3091-2388

Ho Kim, https://orcid.org/0000-0001-7472-3752

Jung Pyo Lee, https://orcid.org/0000-0002-4714-1260

\section{References}

1. Prüss-Ustün A, Wolf J, Corval án C, Bos R, Neira M. Preventing disease through healthy environments: A global assessment of the burden of disease from environmental risks [Internet]. Geneva: World Health Organization; 2016 [cited 2021 Apr 15]. Available from: https://apps.who.int/iris/handle/10665/204585.

2. Maas J, Verheij RA, Groenewegen PP, de Vries S, Spreeuwenberg P. Green space, urbanity, and health: how strong is the relation? J Epidemiol Community Health 2006;60:587-592.

3. Hystad P, Davies HW, Frank L, et al. Residential greenness and birth outcomes: evaluating the influence of spatially correlated built-environment factors. Environ Health Perspect 2014;122:1095-1102.

4. Coresh J, Selvin E, Stevens LA, et al. Prevalence of chronic kidney disease in the United States. JAMA 2007;298:2038-2047.

5. Honeycutt AA, Segel JE, Zhuo X, Hoerger TJ, Imai K, Williams D. Medical costs of CKD in the Medicare population. J Am Soc Nephrol 2013;24:1478-1483.

6. Couser WG, Remuzzi G, Mendis S, Tonelli M. The contribution of chronic kidney disease to the global burden of major noncommunicable diseases. Kidney Int 2011;80:1258-1270.

7. Agarwal R. The challenge of discovering patient-level cardiovascular risk factors in chronic kidney disease. Kidney Int 2008;73:1340-1342.

8. Yoon UA, Kim YC, Lee H, et al. The impact of sunlight exposure on mortality of patients with end stage renal disease. Sci Rep 2019;9:2230

9. Levin A, Stevens PE, Bilous RW, et al. Kidney Disease: Improv- ing Global Outcomes (KDIGO) CKD Work Group. KDIGO 2012 clinical practice guideline for the evaluation and management of chronic kidney disease. Kidney Int Suppl 2013;3:1-150.

10. Didan K. MOD13Q1 MODIS/Terra vegetation indices 16-day L3 global 250m SIN grid V006 [Internet]. NASA EOSDIS Land Processes DAAC; 2015 [cited 2021 Apr 15]. Available from: https:// doi.org/10.5067/MODIS/MOD13Q1.006.

11. Villeneuve PJ, Jerrett M, Su JG, et al. A cohort study relating urban green space with mortality in Ontario, Canada. Environ Res 2012;115:51-58.

12. Pettorelli N, Vik JO, Mysterud A, Gaillard JM, Tucker CJ, Stenseth NC. Using the satellite-derived NDVI to assess ecological responses to environmental change. Trends Ecol Evol 2005;20:503-510.

13. Turvey CG, Mclaurin MK. Applicability of the normalized difference vegetation index (NDVI) in index-based crop insurance design. Weather Clim Soc 2012;4:271-284.

14. Thiering E, Markevych I, Brüske I, et al. Associations of residential long-term air pollution exposures and satellite-derived greenness with insulin resistance in german adolescents. Environ Health Perspect 2016;124:1291-1298.

15. James P, Hart JE, Banay RF, Laden F. Exposure to greenness and mortality in a nationwide prospective cohort study of women. Environ Health Perspect 2016;124:1344-1352.

16. Chudleigh RA, Ollerton RL, Dunseath G, et al. Performance of the revised '175' Modification of Diet in Renal Disease equation in patients with type 2 diabetes. Diabetologia 2008;51:17141718.

17. Crouse DL, Pinault L, Balram A, et al. Urban greenness and mortality in Canada's largest cities: a national cohort study. Lancet Planet Health 2017;1:e289.

18. Kihal-Talantikite W, Padilla CM, Lalloué B, et al. Green space, social inequalities and neonatal mortality in France. BMC Pregnancy Childbirth 2013;13:191.

19. Dadvand P, Sunyer J, Basagaña X, et al. Surrounding greenness and pregnancy outcomes in four Spanish birth cohorts. Environ Health Perspect 2012;120:1481-1487.

20. Gascon M, Triguero-Mas M, Martínez D, et al. Residential green spaces and mortality: a systematic review. Environ Int 2016;86:60-67.

21. Bodicoat DH, O'Donovan G, Dalton AM, et al. The association between neighbourhood greenspace and type 2 diabetes in a large cross-sectional study. BMJ Open 2014;4:e006076.

22. Hirabayashi S, Nowak DJ. Comprehensive national database of tree effects on air quality and human health in the United States. Environ Pollut 2016;215:48-57. 
23. Kim HJ, Min JY, Kim HJ, Min KB. Parks and green areas are associated with decreased risk for hyperlipidemia. Int J Environ Res Public Health 2016;13:1205.

24. Yang J, McBride J, Zhou J, Sun Z. The urban forest in Beijing and its role in air pollution reduction. Urban For Urban Green 2005;3:65-78.

25. Eze IC, Hemkens LG, Bucher HC, et al. Association between ambient air pollution and diabetes mellitus in Europe and North America: systematic review and meta-analysis. Environ Health Perspect 2015;123:381-389.

26. Yang BY, Qian Z, Howard SW, et al. Global association between ambient air pollution and blood pressure: a systematic review and meta-analysis. Environ Pollut 2018;235:576-588.

27. Rook GA. Regulation of the immune system by biodiversity from the natural environment: an ecosystem service essential to health. Proc Natl Acad Sci U S A 2013;110:18360-18367.

28. Jia X, Yu Y, Xia W, et al. Cardiovascular diseases in middle aged and older adults in China: the joint effects and mediation of different types of physical exercise and neighborhood greenness and walkability. Environ Res 2018;167:175-183.

29. Yang BY, Liu KK, Markevych I, et al. Association between residential greenness and metabolic syndrome in Chinese adults. Environ Int 2020;135:105388.

30. Clegg A, Young J, Iliffe S, Rikkert MO, Rockwood K. Frailty in elderly people. Lancet 2013;381:752-762.

31. Orioli R, Antonucci C, Scortichini M, et al. Exposure to residential greenness as a predictor of cause-specific mortality and stroke incidence in the rome longitudinal study. Environ Health Perspect 2019;127:27002.

32. Zhu A, Yan LL, Wu CD, James P, Zeng Y, Ji JS. Residential greenness, activities of daily living, and instrumental activities of daily living: a longitudinal cohort study of older adults in China. Environ Epidemiol 2019;3:e065. 